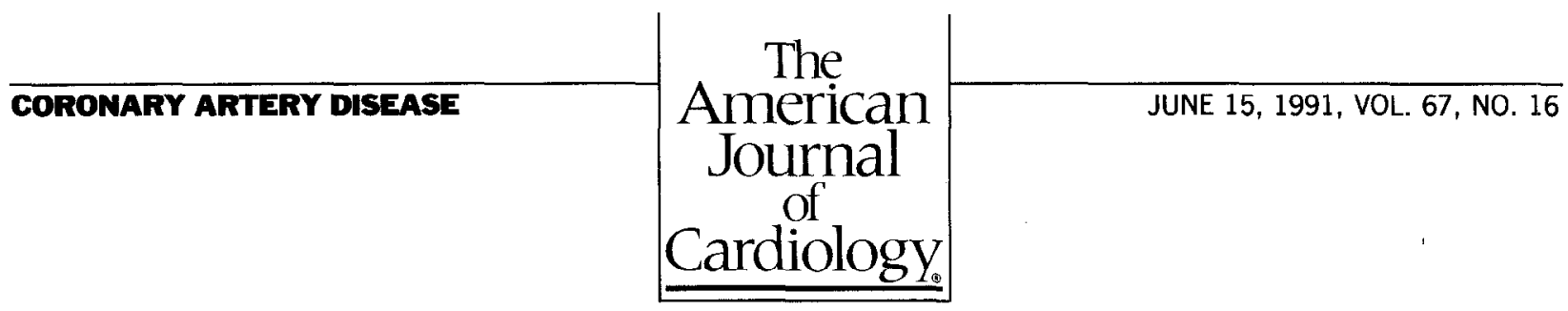

\title{
Comparison of Rubidium-82 Positron Emission Tomography and Thallium-201 SPECT Imaging for Detection of Coronary Artery Disease
}

\author{
Richard E. Stewart, MD , Markus Schwaiger, MD, Edgar Molina, BS, Jeffrey Popma, MD, \\ Gerald M. Gacioch, MD, Morton Kalus, MD, Sheila Squicciarini, BS, RT(N), \\ Ziad R. Al-Aouar, MD, Anthony Schork, PhD, and David E. Kuhl, MD
}

The diagnostic performance of rubidium- 82 (Rb82) positron emission tomography (PET) and thallium-201 (TI-201) single-photon emissioncomputed tomography (SPECT) for detecting coronary artery disease was investigated in 81 patients (52 men, 29 women). PET studies using $60 \mathrm{mCl}$ of Rb-82 were performed at baseline and after intravenous infusion of $0.56 \mathrm{mg} / \mathrm{kg}$ dipyridamole in conjunction with handgrip stress. TI-201 SPECT was performed after dipyridamole-handgrip stress and, In a subset of patients, after treadmill exercise. Sensitivity, specificity and overall diagnostic accuracy were assessed using both visually and quantitatively interpreted coronary angiograms. The overall sensitivity, specificity and accuracy of PET for detection of coronary artery disease $(>\mathbf{5 0} \% \mathrm{di}-$ ameter stenosis) were 84,88 and $85 \%$, respectively. In comparison, the performance of SPECT revealed a sensitivity of $84 \%$, specificity of $53 \%$ (p $<0.05$ vs PET) and accuracy of $79 \%$. Similar results were obtained using either visual or quantitative angiographic criteria for severity of coronary artery disease. In $\mathbf{4 3}$ patients without

From the University of Michigan Medical Center, Department of Internal Medicine, Division of Nuclear Medicine, Ann Arbor, Michigan. This study was performed during the tenure of an established investigatorship from the American Heart Association, Dallas, Texas (M. Schwaiger), and was also supported in part by Grant RO1 HL41047-01 from the National Institutes of Health. Bethesda, Maryland. The Rubidium-82 generators used for this study were supplied by Squibb Diagnostics Inc., Princeton, New Jersey. Manuscript received October 26, 1990; revised manuscript received and accepted February $19,1991$.

Address for reprints: Markus Schwaiger, MD, University of Michigan Mcdical Center, 1500 E. Mcdical Center Drive; UH B1 G505/ 0028, Ann Arbor, Michigan 48109-0028. prior myocardial infarction, the sensitivity for detection of disease was 71 and $73 \%$, respectively, similar for both PET and SPECT. There was no significant difference in diagnostic performance between imaging modalities when 2 different modes of stress (exercise treadmill vs intravenous dipyridamole plus handgrip) were used with SPECT imaging. Thus, Rb-82 PET provides improved specificity compared with TI201 SPECT for identifying coronary artery disease, most llkely due to the higher photon ener.. gy of Rb-82 and attenuation correction provided by PET. However, post-test referral cannot be entirely excluded as a potential explanation for the lower specificity of TI-201 SPECT.

(Am J Cardiol 1991;67:1303-1310)

$\mathrm{E}$ arly detection of coronary artery disease by noninvasive techniques remains a major clinical goal in cardiology. Stress imaging with markers of myocardial perfusion has emerged as the preferred approach in patients suspected of having coronary artery disease. ${ }^{1,2}$ In combination with advanced imaging technology such as single-photon emission-computed tomography (SPECT), thallium-201 (Tl-201) or technetium-99m-labeled compounds have proven accurate in the sensitive detection and localization of coronary artery disease. ${ }^{3-6}$ However, SPECT does not allow for correction of photon attenuation, which leads frequently to artifactual tracer uptake abnormalities, limiting the specificity of the test. ${ }^{7,8}$

Positron emission tomography (PET) represents a considerable technologic improvement compared with conventional single-photon tomography. Because of 
the unique data acquisition, regional myocardial tracer retention can be corrected for photon attenuation yielding quantitative assessment of regional activity concentration. $^{9}$ The potassium analog rubidium- 82 $(\mathrm{Rb}-82)$, which is a generator-produced radioisotope, has emerged as the most practical PET flow tracer. $\mathrm{Rb}-82$ production by a commercially available generator system avoids the need for isotope production by an on-sitc cyclotron. ${ }^{10}$ Previous studies using $\mathrm{Rb}-82$ or $\mathrm{N}-13$ ammonia in combination with PET have shown high diagnostic accuracy in detecting coronary artery disease. ${ }^{11-13}$ However, these studies did not include the direct comparison of Rb-82 PET with Tl-201 SPECT in the same patient population. Thus, the purpose of this study was to evaluate both techniques in the same patient population and to compare the imaging results with those of coronary angiography.

\section{METHODS}

Patients: Patients referred to the University of Michigan Medical Center for diagnostic coronary angiography were selected prospectively. The referral diagnosis included chest pain, history of infarct, presurgical evaluation and abnormal stress test. Patients with previous coronary bypass surgery or percutaneous transluminal coronary angioplasty were excluded. In addition, patients with a low likelihood of coronary artery disease based on age, sex and risk factors (absence of hypertension, diabetes mellitus, hypercholesterolemia, smoking history or family history), who did not undergo coronary arteriography, were selected from a patient population undergoing Tl-201 SPECT stress testing in the Division of Nuclear Medicine at the University of Michigan. All patients underwent Tl-201 SPECT and $\mathrm{Rb}-82$ PET imaging within a time period of 3 weeks. Patients with changing clinical status between both tests were excluded.

Thallium-201 single-photon emission-computed tomography imaging: T1-201 SPECT imaging was performed in combination with either exercise testing or intravenous dipyridamole infusion. All patients fasted for $\geq 8$ hours before the test. In patients undergoing excrcise stress testing, the standard Bruce protocol was used. At maximal exercise, $3 \mathrm{mCi}$ of Tl-201 was injected intravenously and exercise continued for 1 minute. SPECT acquisition began between 10 and 15 minutes after the end of exercise and was completed within 45 minutes after tracer injection.

In patients undergoing pharmacologic stress testing, dipyridamole $(0.56 \mathrm{mg} / \mathrm{kg})$ was infused intravenously over 4 minutes. Four minutes after the end of the dipyridamole infusion, the patient was asked to perform handgrip exercise (30\% of maximal strength). ${ }^{14}$ One minute after initiation of handgrip exercise, $3 \mathrm{mCi}$ of
Tl-201 was injected intravenously, and handgrip exercise was maintained for another 2 minutes. Imaging was started 10 minutes after the injection of Tl-201 using the same protocol as that after exercise testing (Figure 1).

DATA ACQUISITION: Image acquisition was performed using a General Electric 400AC gamma camera system with a parallel-hole, low-energy, all-purpose collimator. This camera system was interlinked to a MicroDelta workstation (Siemens, Knoxville, Tennessee). Data were acquired over a $180^{\circ}$ anglc starting from the $45^{\circ}$ right anterior oblique projection. Data collection consisted of 64 steps of 15 seconds each. All patients underwent redistribution imaging 4 to 6 hours after the initial tracer injection.

IMAGE PROCESSING: Image processing consisted of filtered back projection of data in a $64 \times 64$ word matrix and reconstruction of transverse images using a Butterworth filter with a cutoff frequency of 0.35 cycles/pixel. The tomographic images were subsequently realigned perpendicular to the long axis of the left ventricle using commercially available software (MicroDelta workstation). All images were displayed in black and white using a large screen display system (DeltaVision, CDA) and customized software, which allows the direct comparison of stress and redistribution images in oblique, longitudinal and sagittal views.

DATA ANALYSIS: All images were cvaluatcd by 2 observers blinded to clinical and PET data using a visual scoring system. In case of disagreement, a third observer graded the images. One represented normal, 2 equivocal, 3 mildly abnormal and 4 markedly abnormal. Visual assessment was performed in 4 sections in each of 3 left ventricular slices (apical, mid and basal). Each of these 3 slides was divided into anterior, lateral, inferior and septal segments for a total of 12 scoring segments for each study. Stress and redistribution images were evaluated using the same scoring system. An abnormal scan was defined as a Tl-201 defect score of $\geq 3$ in at least 1 of the 12 segments.

Positron emission tomography: All patients were studied after an overnight fast using a 15-slice whole body PET scanner (931 CTI/Siemens, Knoxville, Tennessee). After positioning of the patient in the gantry, a transmission scan using a retractable Germanium-68 ring source was performed for 15 to 20 minutes ( 100 to 200 million counts). The transmission scan was followed by a baseline Rb-82 study, which consisted of the infusion of $60 \mathrm{mCi}$ of $\mathrm{Rb}-82$ using the strontium82/Rb-82 generator (Squibb Diagnostic, Inc., Princeton, New Jersey) and infusion system (CTI). Rb-82 was infused with a pump setting of $50 \mathrm{ml} / \mathrm{min}$ and a specific activity of 1 to $2 \mathrm{mCi} / \mathrm{ml}$. Sixty seconds after the end of Rb-82 infusion, data were acquired for 7 
minutes. Fifteen minutes after the studies at rest, an intravenous infusion of dipyridamole $(0.56 \mathrm{mg} / \mathrm{kg})$ was started for 4 minutes. As stated, 4 minutes after the end of the dipyridamole infusion, handgrip exercise was initiated, and 1 minute later, $60 \mathrm{mCi}$ of $\mathrm{Rb}-82$ was infused using the same protocol. For graphic display of the imaging protocol, see Figure 1.

IMAGE PROCESSING: The attenuation-corrected projection data were used to reconstruct transverse images with a Hanning filter and a cutoff frequency of 0.35 cycles/pixels. Using a computer workstation (SUN systems) and dedicated software (volume tool, CTI), the transverse images were realigned perpendicular to the long axis of the left ventricle yielding cross-sectional and longitudinal images.

DATA ANALYSIS: The cross-sectional images were displayed in the same format as the Tl-201 SPECT images. Regional tracer retention at baseline and after stress was evaluated by 2 observers unaware of the clinical information and Tl-201 SPECT data. In the case of disagreement, a third observer was consulted. PET studies were read in similar orientation as Tl-201 SPECT. The left ventricle was divided into 12 sectors ( 4 sections in each of 3 ventricular slices from apex to base), and regional $\mathrm{Rb}-82$ retention was graded as previously described for Tl-201 SPECT.
Coronary angiography: Cineangiograms of the coronary arteries were obtained in multiple projections using a Siemens C-Core angiographic system (5-inch intensifier, image resolution 3.8 line pair $/ \mathrm{mm}$ ). The following angiographic criteria were used to define the presence of a significant coronary stenosis: (1) visually determined diameter stenosis $\geq 50 \%$, (2) visually determined diameter stenosis $\geq 70 \%$, (3) quantitatively determincd diameter stenosis $\geq 50 \%$, (4) quantitatively determined percent area stenosis $\geq 70 \%$, or (5) minimal cross-sectional area $<2.0 \mathrm{~mm}^{2}$ in proximal or midepicardial vessel..$^{15}$ Eighty-two percent of the angiograms were of sufficient quality to perform quantitative analysis.

STATISTICAL ANALYSIS: Data were expressed in terms of sensitivity and specificity as well as diagnostic accuracy, with the results of coronary angiography serving as the gold standard. Statistical analysis involved the McNemar chi-square test for differences in group data. A p value $<0.05$ was considered statistically significant.

\section{RESULTS}

Patient characteristics: Eighty-one patients were selected consecutively from inpatient and outpatient populations over a 12-month period. The study included 52 men and 29 women (mean age \pm standard deviation 57

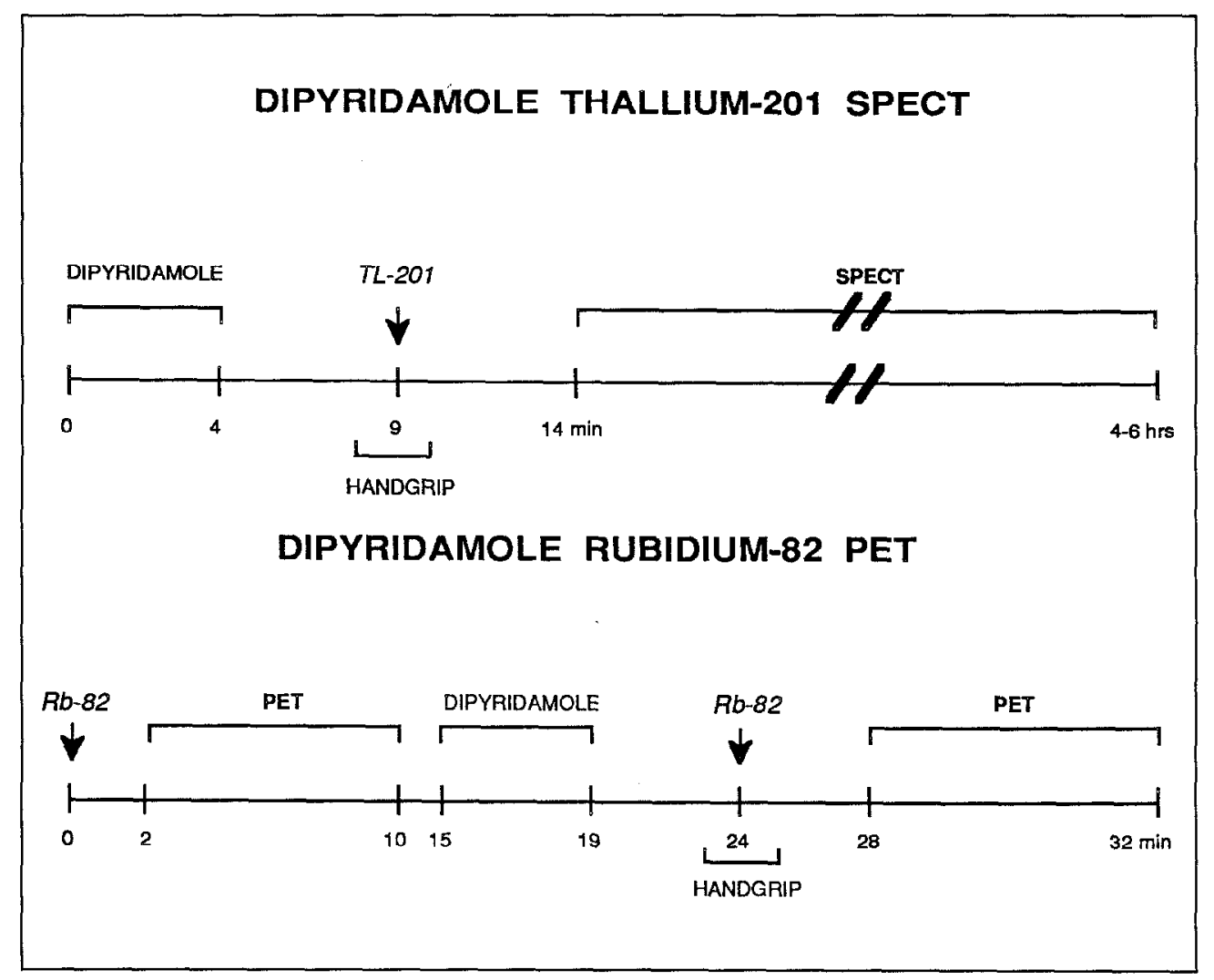

FICURE 1. Imaging protocol for thalilum-201 (TL-201) single-photon emission-computed tomography (SPECT) and rubidium$82($ Rb-82) positron emission tomography (PET) dipyridamole studies. 
\pm 12 years). Ninety-three percent of the patients underwent coronary angiography, whereas 6 patients (4 women, 2 men) were selected because of low likelihood of having coronary artery disease. Based on the visual assessment of coronary angiography, 60 of 81 patients had significant coronary artery disease ( $>50 \%$ stenosis) involving at least 1 major coronary artery. Thirty-four patients with coronary artery disease had clinical, electrocardiographic or enzymatic evidence of prior history of myocardial infarction. Twenty-one patients were considered free of significant coronary artery disease by angiographic criteria. Of those patients with significant disease, 20 patients had 1 -vessel, 27 had 2-vessel and 13 had 3-vessel disease. Fifty-eight percent of patients underwent coronary angiography before the imaging procedures.

Stress testing: All patients underwent dipyridamole infusion in combination with PET imaging. Thirtyeight patients underwent exercise T1-201 SPECT. The double product achieved averaged 26,314 \pm 3.077 . Forty-three patients underwent dipyridamole Tl-201 SPECT.

Scintigraphic results: The administration of $60 \mathrm{mCi}$ of $\mathrm{Rb}-82$ resulted in 400,000 to 600,000 counts (true coincidence event) per plane for 15 image planes. The image quality was considered good or excellent in $87 \%$ of the patients as characterized by clear delineation of left ventricular myocardium. The administration of $3 \mathrm{mCi}$ of T1-201 resulted in 200,000 to 300,000 counts per SPECT plane. Ninety-five percent of Tl-201 SPECT studies were judged good or excellent. All studies were included in the data analysis. Figures 2 and 3 compare Rb-82 PET and Tl-201 SPECT studies in 2 patients with significant coronary artery disease involving the left anterior descending and right coronary arteries, respectively. Both image sets were displayed and photographed using the same workstation. Regional tracer uptake was decreased in the affected vascular territories in both the PET and SPECT studies. However, the perfusion defects appear more clearly defined on the PET images, most likely as a result of increased spatial resolution provided by this technique.

Overall diagnostic performance: The overall diagnostic performance of both imaging methods is summarized in Table I. The scintigraphic results were related to the anatomic severity and extent of coronary artery disease assessed by visual and quantitative analysis of coronary angiograms. The sensitivity for detecting coro-
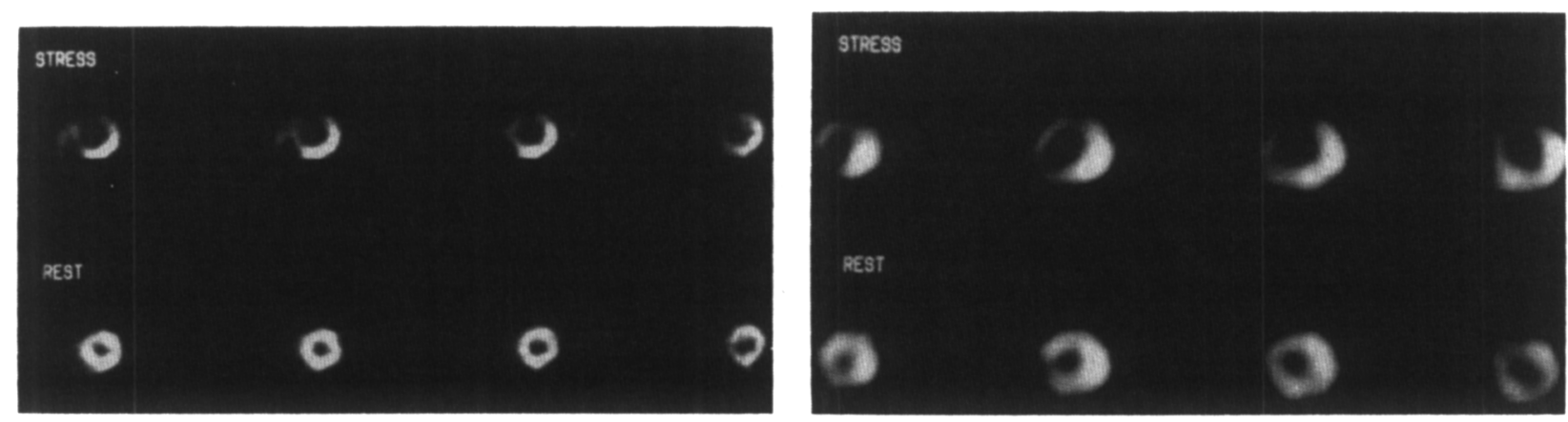

FIGURE 2. Rubidium-82 (Rb-82) positron emission tomography (left) and thallium-201 (n-201) single-photon emission-computed tomography (right) study in a patient with significant disease involving the left anterior descending artery. Four short-axis images after stress (above) are compared with resting Rb-82 or delayed $\mathbf{7 - 2 0 1}$ images (below). In both studies there is a significant decrease of the perfusion defect under resting and delayed conditions consistent with the clinical history of no prior myocardial infarction in this patient.
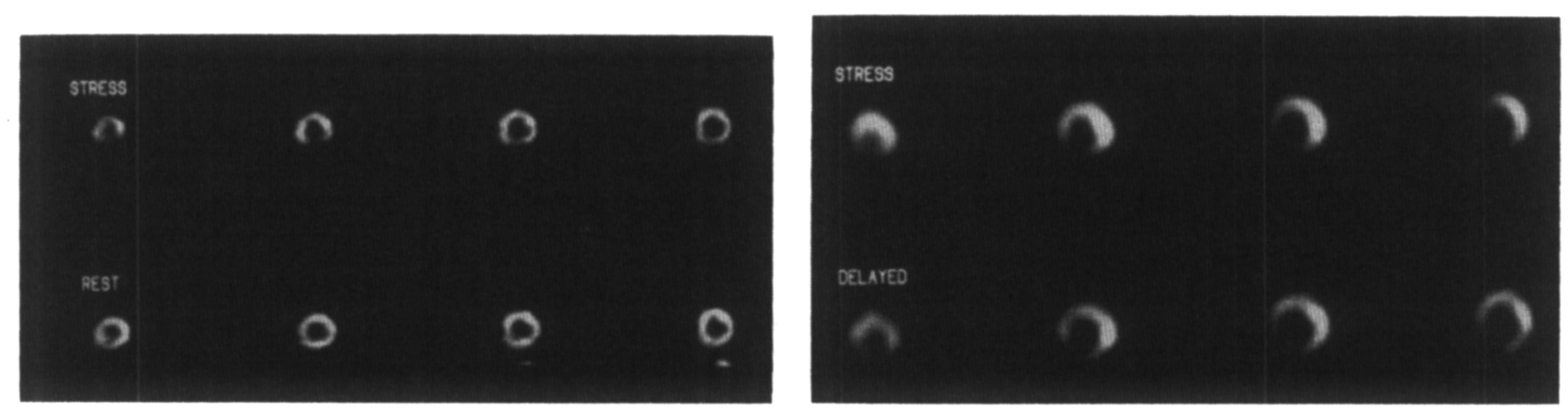

FIGURE 3. Rubidium-82 (Rb-82) positron emission tomography (left) and thallium-201 (T-201) single-photon emission-computed tomography (right) study in a patient with significant coronary artery disease (90\% stenosis) in the right coronary artery without history of previous infarction. Note lack of redistribution on the 7-201 study in contrast to the normal resting Rb-82 study in this patient without previous infarction. 


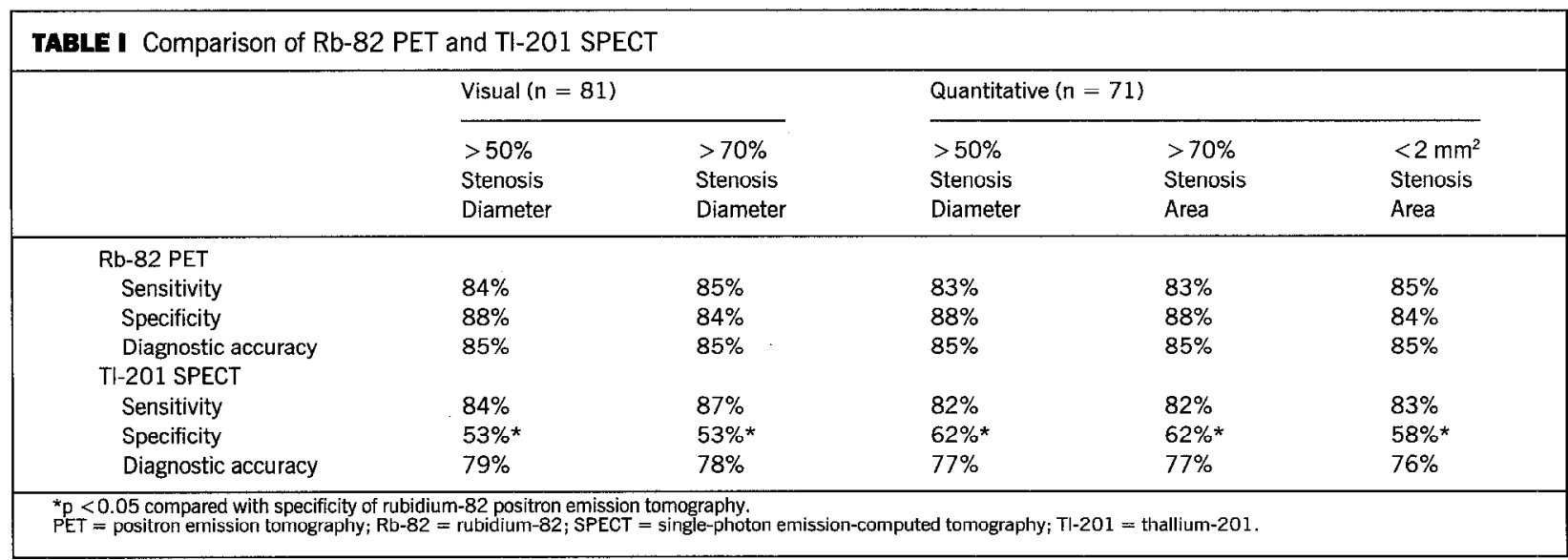

\begin{tabular}{|c|c|c|c|c|c|}
\hline & \multicolumn{2}{|c|}{ Visual $(n=43)$} & \multicolumn{3}{|c|}{ Quantitative $(n=41)$} \\
\hline & $\begin{array}{l}>50 \% \\
\text { Stenosis } \\
\text { Diameter }\end{array}$ & $\begin{array}{l}>70 \% \\
\text { Stenosis } \\
\text { Diameter }\end{array}$ & $\begin{array}{l}>50 \% \\
\text { Stenosis } \\
\text { Diameter }\end{array}$ & $\begin{array}{l}>70 \% \\
\text { Stenosis } \\
\text { Area }\end{array}$ & $\begin{array}{l}<2 \mathrm{~mm}^{2} \\
\text { Stenosis } \\
\text { Area }\end{array}$ \\
\hline \multicolumn{6}{|l|}{ Rb-82 PET } \\
\hline Sensitivity & $71 \%$ & $71 \%$ & $75 \%$ & $75 \%$ & $73 \%$ \\
\hline Specificity & $88 \%$ & $84 \%$ & $88 \%$ & $88 \%$ & $84 \%$ \\
\hline Diagnostic accuracy & $77 \%$ & $77 \%$ & $80 \%$ & $80 \%$ & $78 \%$ \\
\hline \multicolumn{6}{|l|}{ TI-201 SPECI } \\
\hline Sensitivity & $73 \%$ & $75 \%$ & $72 \%$ & $72 \%$ & $73 \%$ \\
\hline Specificity & $53 \% *$ & $53 \% *$ & $62 \% *$ & $62 \% *$ & $58 \% *$ \\
\hline Diagnostic accuracy & $65 \%$ & $6 b \%$ & $68 \%$ & $68 \%$ & $60 \%$ \\
\hline
\end{tabular}

nary artery disease in 60 patients was not different for $\mathrm{Rb}-82$ PET or Tl-201 SPECT. The specificity of $\mathrm{Rb}-$ 82 PET was significantly greater $(\mathrm{p}<0.05)$ compared with that of Tl-201 SPECT. The sensitivity values of both tests were similar for all angiographic criteria (Table I). However, the specificity was consistently higher for Rb-82 PET imaging compared with T1-201 SPECT. Quantitative analysis of coronary angiography yielded a higher specificity compared with visual analysis, which was not statistically significant.

Figure 4 shows an example of improved diagnostic accuracy of Rb-82 PET. Hour cross-sectional Rb-82 and Tl-201 images obtained in a patient with angiographically normal coronary arteries are shown. The stress Tl-201 study revealed decreased activity in the inferior wall, whereas the regional tracer distribution was homogeneous in the corresponding stress $\mathrm{Rb}-82$ PET study.

Diagnostic performance in patients with and without prior myocardial infarction: In 43 patients without prior myocardial infarction, the sensitivity for detecting coronary artery disease was $71 \%$ for Rb-82 PET and $73 \%$ for Tl-201 SPECT (based on $>50 \%$ diameter stenosis) (Table II).
Both tests performed equally in detecting previous myocardial infarction. The frequency of an abnormal T1-201 SPECT or Rb-82 PET study was $97 \%$ in patients with previous myocardial infarction.

Comparison of exercise versus dipyridamole stress: Because not all patients underwent dipyridamole Tl201 SPECT, the diagnostic performance of both tests was compared in 38 patients undergoing exercise Tl201 SPECT. The sensitivity of exercise Tl-201 SPECT (>50\% diameter stenosis) was $93 \%$ compared with

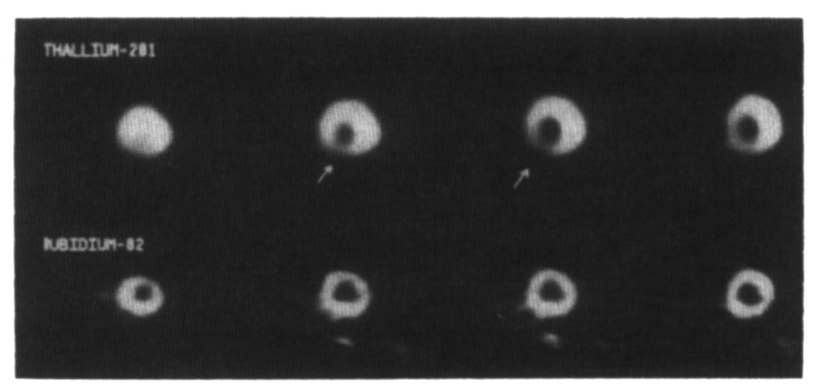

FIGURE 4. Four midventricular short-axis stress images in a patient with normal coronary arteries. The thallium-201 sin. gle-photon emission-computed tomography stress images (above) were read as abnormal in the inferior wall, whereas the rubidium-82 positron emission tomography images (below) were considered normal. 
TABLE III Comparison of Rb-82 PET and TI-201 SPECT

\begin{tabular}{|c|c|c|c|c|c|c|}
\hline & \multicolumn{3}{|c|}{ Exercise SPECT $(n=38)$} & \multicolumn{3}{|c|}{ Dipyridamole SPECT $(n=43)$} \\
\hline & $\begin{array}{l}\text { Sen } \\
(\%)\end{array}$ & $\begin{array}{l}\text { Spec } \\
(\%)\end{array}$ & $\begin{array}{l}\text { Acc } \\
(\%)\end{array}$ & $\begin{array}{l}\text { Sen } \\
(\%)\end{array}$ & $\begin{array}{l}\text { Spec } \\
(\%)\end{array}$ & $\begin{array}{l}\text { Acc } \\
(\%)\end{array}$ \\
\hline Tl-201 SPECT & 92 & 55 & 82 & 82 & 50 & 74 \\
\hline Rb-82 PET & 89 & 90 & 89 & 84 & 73 & 81 \\
\hline
\end{tabular}

\begin{tabular}{|c|c|c|c|c|c|}
\hline & $\begin{array}{l}\text { Septum } \\
(\%)\end{array}$ & $\begin{array}{l}\text { Anterior } \\
(\%)\end{array}$ & $\begin{array}{l}\text { Inferior } \\
(\%)\end{array}$ & $\begin{array}{l}\text { Lateral } \\
(\%)\end{array}$ & $\begin{array}{l}\text { All } \\
\text { (\%) }\end{array}$ \\
\hline Rb-82 PET & 87 & 92 & 94 & 89 & 91 \\
\hline T1-201 SPECT & 89 & 98 & 92 & 90 & 95 \\
\hline
\end{tabular}

$89 \%$ for $\mathrm{Rb}-82 \mathrm{PET}$. The specificity of Rb-82 was 90 vs $55 \%$ for Tl-201 SPECT (Table III).

Forty-three patients underwent dipyridamole stress testing combined with both Rb-82 PET and Tl-201 SPECT. The sensitivity for detecting significant coronary artery disease in this subgroup was 84 and $82 \%$, respectively, with a specificity of $73 \%$ for Rb-82 PET and 50\% for Tl-201 SPECT (Table III).

Interobserver variability: For $91 \%$ of patients, the principal readers (M.S., M.K.) agreed on the overall interpretation of the presence (visual score $\geq 3$ ) or absence (score <3) of Rb-82 PET perfusion defects. Likewise, agreement was reached in $95 \%$ of patients in grading T1-201 SPECT studies. Disagreement most often involved the lateral wall (11\% with PET vs $10 \%$ with SPECT) and septum (13 vs $11 \%$ ) with both studies (Table IV).

\section{DISCUSSION}

The results of this study indicate improved specificity of Rb-82 PET imaging compared with Tl-201 SPECT for detecting coronary artery disease in a selected patient population undergoing coronary angiography. The sensitivity of both tests for identifying significant stenosis was comparable. The enhanced specificity was not influenced by the stress approach (dipyridamole vs exercise). Thus, Rb-82 PET appears to reduce the incidence of false-positive results, most likely because of the improved imaging technology provided by this new imaging technique.

Sensitivity of positron emission tomography versus single-photon emission-computed tomography! The reported sensitivity for Tl-201 SPECT in this study agrees closely with previous reported values for this test and confirms the high diagnostic yield of this procedure. ${ }^{16,17}$ The sensitivity of Rb-82 PET imaging for identifying coronary artery disease in the studied patient population was not different from that of Tl-201 SPECT. Previous studies using PET, in combination with $\mathrm{Rb}-82$ or $\mathrm{N}-13$ ammonia, reported sensitivity values exceeding $90 \% .{ }^{11,12,18}$ This discrepancy may be explained partly by patient selection yielding a higher incidence of advanced coronary artery disease in these studies or by varialion in the analysis of coronary angiography. Demer et al ${ }^{12}$ reported a sensitivity of $94 \%$ for detecting significant coronary stenosis in 193 patients studied with Rb-82 or N-13 ammonia. This study compared the PET results with calculated coronary reserve values, which were based not only on diameter stenosis, but also on the geometry of a given lesion. ${ }^{19}$ Significant coronary artery disease was defined as a coronary reserve value $<3$. If patients with intermediate severity of stenosis (coronary reserve 3 to 4 ) were included in the analysis, the sensitivity for identification of disease by PET decreased to $83 \%$. Therefore, the overall reported sensitivity of Rb-82 PET imaging in this study, ranging from 85 to $88 \%$, may reflect a very similar diagnostic performance than that observed in the study by Demer et al. ${ }^{12}$

The incidence of false-negative Rb-82 PET studies, especially in patients without prior myocardial infarction, may be related to the pharmacologic stress approach. $\Lambda$ recent report by Rossen et $\mathrm{al}^{20}$ indicates the possibility of an attenuated flow response to this pharmacologic stress in about $20 \%$ of patients. Although care was taken to exclude patients taking medications known to abolish the dipyridamole effect, a variable response to dipyridamole in our patient population cannot be entirely excluded as cause for false-negative PET and SPECT studies. In the context of this discussion, it is interesting to note that the sensitivity of Tl-201 exercise testing tended to be higher than that of dipyrida- 
mole Tl-201 SPECT or dipyridamole Rb-82 PET imaging. High-dose dipyridamole or adenosine infusion may enhance coronary vasodilation and, hence, increase the sensitivity of pharmacologic approaches to detect coronary artery disease. ${ }^{21}$

Specificity of rubidium-82 positron emission tomography versus thallium-201 single-photon emission-computed tomography: The specificity for Tl-201 SPECT observed in our study was lower than initially reported for this procedure., ${ }^{4,21}$ Several recent studies comparing the results of Tl-201 SPECT with those of coronary angiography indicate specificity values ranging from 47 to $71 \% ., 16,22,23$ Iskandrian et a ${ }^{16}$ reported an incidence of 22 false-positive Tl-201 SPECT studies in 58 patients with angiographically normal coronary arteries (specificity $62 \%$ ). Using quantitative analysis of Tl-201 SPECT images, Maddahi et al $^{22}$ described a specificity of only $56 \%$ in 18 patients with normal coronary angiograms. ${ }^{22}$ In contrast, both studies reported a higher "normalcy rate" of Tl-201 SPECT $(93 \%, 86 \%)$ in a group of patients with low likelihood of coronary artery disease, but without coronary angiography. 3,22 The specificity of Tl-201 SPECT reported in this study is in close agreement with the results published by these investigators.

Because definition of sensitivity and specificity requires coronary angiography as a gold standard, a selection bias based on the results of the noninvasive stress testing has to be considered. ${ }^{24,25}$ Patients with positive test results will be predominantly referred for cardiac catheterization, whereas few patients with a normal stress test will undergo invasive diagnostic procedures. Diamond ${ }^{24}$ recently discussed the effect of patient referral on determining the diagnostic accuracy of a given test. The goal of this study, however, was not to assess the overall diagnostic performance of each imaging modality, but to compare both tests with angiographic results in the same patient population. The patient population selected in this study may have a higher incidence of "false-positive" Tl-201 SPECT findings compared with a patient cohort with low likelihood of disease. However, the improved specificity of Rb-82 PET may demonstrate the superiority of PET imaging in patients prone to have attenuation artifacts on Tl201 SPECT images. Patients with low likelihood of coronary artery disease tend to be younger, less obese and tolerate higher exercise levels. Such a selected control group is quite different from the typical patient population suspected of having coronary artery disease, and the normalcy rate may not reflect the true specificity of the test. ${ }^{26,27}$

The greater specificity of Rb-82 PET imaging may be important in the case of Tl-201 defects involving the inferior wall (Figure 4). The increased incidence of false-positive Tl-201 SPECT perfusion defects in this region is well recognized and most likely represents attenuation artifacts involving the diaphragm. ${ }^{7,8,28,29}$ PET provides attenuation correction and, thus, is expected to be less affected by diaphragmatic attenuation. ${ }^{9}$ Recently, SPECT imaging in the prone position has been advocated to reduce attenuation artifacts in the male patient population. ${ }^{29}$ Although first results are promising, no data are currently available to document the improved specificity of this imaging approach.

Advantages of rubidium-82 as a perfusion tracerı Besides the improved specificity of PET in combination with $\mathrm{Rb}-82$, there are several additional advantages of this new imaging approach. Rb- 82 is a generator-produced compound that can be easily administered using a simple infusion system available 24 hours each day. Its short half-life of 76 seconds reduces the radiation exposure to the patient. The time required for a resting and stress blood flow evaluation averages approximately 1 hour for Rb-82 PET, whereas Tl-201 imaging involves a waiting period of several hours between the stress and delayed imaging procedure. Those practical advantages are offset by the higher costs of the PET imaging procedure. However, a recent study indicates that a dedicated Rb-82 PET camera may be financially viable with 8 to 10 fully reimbursed studies per day. ${ }^{30}$

Conclusions: PET, in combination with Rb-82, provides improved specificity in detecting coronary artery disease compared with Tl-201 SPECT. Higher photon energy of Rb-82 and attenuation correction provided by PET may be primarily responsible for this diagnostic gain. Together with the short half-life of the tracer, $\mathrm{Rb}$. 82 PET imaging provides a rapid evaluation of regional coronary reserve in patients suspected of having coronary artery disease. PET, in combination with various flow tracers, may provide clinically important information for accurately identifying and quantifying ischemic heart disease.

Acknowledgment: We appreciate the excellent technical support by Jill Rothley, Leslie Shaw, Vincent McCormick and Annette Bentley in performing the PET studies. Dipyridamole was kindly supplied by Boehinger, Inc. Ingelheim, West Germany. The Rb-82 generators were generously provided by Squibb Inc. and the infusion system by CTI/Siemens. Finally, we thank Vi Rhodes and Tina Bennett for preparing the manuscript.

\section{REFERENCES}

1. Ritchie JL, Trobaugh GB, Hamilton GW, Gould KL. Myocardial imaging with thallium-201 at rest and during exercise. Comparison with coronary arteriography and resting and stress electrocardiography. Circulation 1977;56:56-66. 
2. Baily IK, Griffith LSC, Rouleau J, Strauss W, Pitt B. Tl-201 myocardial perfusion imaging at rest and during exercise: comparative sensitivity to electrocardiography in coronary artery disease. Circulation 1977;55:79-87.

3. Iskandrian AS, Segal BL. Value of exercise Tl-201 imaging in patients with diagnostic and non-diagnostic exercise electrocardiograms. Am J Cardiol 1981; 48:233-238

4. DePasquale EE, Nody AC, DePuey EG, Garcia EV, Pilcher G, Bredleau C, Roubin G, Gober A, Gruentzig A, D'Amato P, Berger HJ. Quantitative rotational thallium-201 tomography for identifying and localizing coronary artery disease. Circulation 1988;77:316-327.

5. Garcia EV, VanTrain K, Maddahi J, Prigent F, Friedman J, Areeda J, Waxman A, Berman DS. Quantification of rotational thallium-201 myocardial tomography. J Nucl Med 1985;26:17-26.

6. Kiat H, Maddahi J, Ray LT, VanTrain K, Friedman J, Resser K, Berman DS. Comparison of Tc-99m methoxy isobutyl isonitril and thallium-201 for evaluation of coronary artery disease by planar and tomographic methods. Am Heart $J$ 1989;1 17:1-11.

7. Friedman J, VanTrain K, Maddahi J, Rozanski A, Prigent F, Bietendorf J, Waxman A, Berman DS. "Upward Creep" of the heart: a frequent source of falsepositive reversible defects during thallium-201 stress-redistribution SPECT. $J$ Nucl Med 1989;30:1718 1722.

8. Gordon DG, Pfisterer M, Williams R, Walaski S, Asburn W. The effect of diaphragmatic attenuation of thallium-201 images. Clin Nucl Med 1979;4: 150-151.

9. Hoffman E, Phelps M. Positron emission tomography. Principles and quantitation. In: Phelps E, Maziotta J, Shelbert H, eds. Positron Emission Tomography and Autoradiography, New York: Raven Press, 198G:237-28G.

10. Yano $Y$, Cahoon JL, Budinger TF. A precision flow controlled Rb-82 generator for bolus or constant-infusion studies of the heart and brain. $J$ Nucl Med 1981;22:1006-1010.

11. Gould KL, Goldstein RA, Mullani NA. Non-invasive assessment of coronary stenoses by myocardial perfusion imaging during pharmacologic coronary vasodilation. VIII. Clinical feasibility of positron cardiac imaging without a cyclotron using generator-produced rubidium-82. J Am Coll Cardiol 1986;7:775-789.

12. Demer LL, Gould KL, Goldstein RA. Assessment of coronary artery disease severity by positron emission tomography. Circulation 1989;79:825-835.

13. Schelbert HR, Wisenberg G, Phelps ME. Non-invasive assessment of coronary stenoses by myocardial imaging during pharmacologic coronary vasodilation. VI. Detection of coronary artery disease in human beings with intravenous N-13 ammonia and positron computed tomography. Am J Cardiol 1982;49: 1197-1207.

14. Brown $G$, Josephson MA, Peterson RB. Intravenous dipyridamole combined with isometric handgrip for near maximal acute increase in coronary flow in patients with coronary artery disease. Am J Cardiol 1981;48:1077-1085.

15. Mancini GBJ, Simon SB, McGillem MJ, LeFree MT, Friedman HZ, Vogel
RA. Automated quantitative coronary arteriography: morphologic and physiologic validation in vivo of a rapid digital angiographic method? Circulation 1987;75:452-460.

16. Iskandrian AS, Heo J, Kong B, Lyons E. Effect of exercise level on the ability of thallium-201 tomographic imaging in detecting coronary artery disease: analysis of 461 patients. J Am Coll Cardiol 1989;14:1477-1486.

17. Maddahi J, Garcia EV, Berman DS, Waxman A, Swan HJC, Forrester J. Improved non-invasive assessment of coronary artery disease by quantitative analysis of regional stress myocardial distribution and washout of thallium-201. Circulation 1981;64:924-935.

18. Go RT, Marwick TH, MacIntyre WJ, Saha GB, Neumann DR, Underwood DA, Simpfendorfer CC. A prospective comparison of rubidium-82 PET and thallium-201 SPECT myocardial perfusion imaging utilizing a single dipyridamole stress in the diagnosis of coronary artery disease. $J \mathrm{Nucl} \mathrm{Med} 1990$; 31:1899-1905.

19. Gould KL, Kirkeeide R. Quantitation of coronary artery stenosis in vivo. Circ Res 1985;57:341-353.

20. Rossen JD, Simonetti I, Marcus ML, Winniford MD. Coronary dilation with standard dose dipyridamole and dipyridamole combined with handgrip. Circulation 1989;79:566-572.

21. Verani MS, Mahmarian JJ, Hixson JB, Boyce TM, Staudachcr RA. Diagnosis of coronary artery disease by controlled coronary vasodilation with adenosine and thallium-201 in patients unable to exercise. Circulation 1990;82:80-87. 22. Maddahi J, Van Train K, Prigent $F$, Garcia EV, Friedman J, Ostrzega E, Berman D. Quantitative single photon emission computed thallium-201 tomography for detection and localization of coronary artery disease: optimization and prospective validation of a new technique. $J$ Am Cull Curdiol 1989;14:1689-1699. 23. Van Train KF, Berman DS, Garcia EV. Quantitative analysis of stress thallium-201 myocardial scintigrams: a multicenter trial. J Nucl Med 1986; 27:17-25.

24. Diamond G. How accurate is SPECT thallium scintigraphy? $J$ Am Coll Cardiol 1990;16:1017-1021.

25. Gould K. Agreement on the accuracy of thallium stress testing. $J$ Am Coll Cardiol 1990;16:1022-1023.

26. Diamond G. An improbable criterion of normality [letter]. Circulation 1982;66:681

27. Diamond G. Monkey business. Am J Cardiol 1986;57:471-475.

28. DePuey EG, Garcia EV. Optimal specificity of thallium-201 SPECT through recognition of imaging artifacts. $J$ Nucl $\mathrm{Med}$ 1989;30:441-449.

29. Esquerre' J-P, Coca FJ, Martinez SJ, Guiraud RF. Prone decubitus: a solution to inferior wall attenuation in thallium-201 myocardial tomography. $J$ Nucl Med 1989;30:398-401.

30. Gould KL, Goldstein RA, Mullani NA. Economic analysis of clinical positron emission tomography of the heart with rubidium-82. J Nucl Med 1989;30: 707-717. 